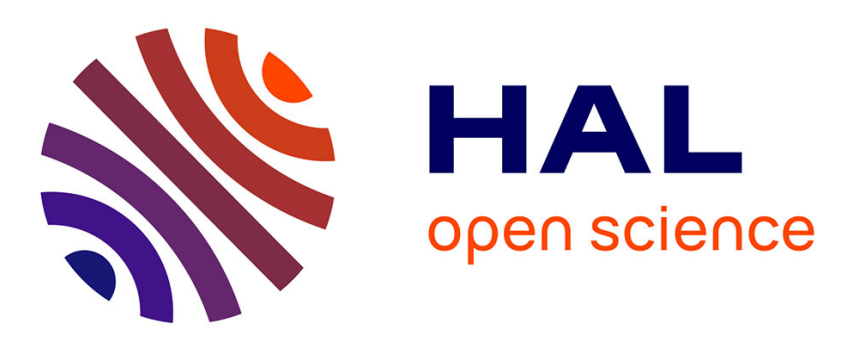

\title{
Fluid of hard spheres with dipolar-like patch interaction and effect of adding an isotropic adhesion
}

Domenico Gazzillo

\section{To cite this version:}

Domenico Gazzillo. Fluid of hard spheres with dipolar-like patch interaction and effect of adding an isotropic adhesion. Molecular Physics, 2010, pp.1. 10.1080/00268976.2010.521780 . hal-00637020

\section{HAL Id: hal-00637020 \\ https://hal.science/hal-00637020}

Submitted on 29 Oct 2011

HAL is a multi-disciplinary open access archive for the deposit and dissemination of scientific research documents, whether they are published or not. The documents may come from teaching and research institutions in France or abroad, or from public or private research centers.
L'archive ouverte pluridisciplinaire HAL, est destinée au dépôt et à la diffusion de documents scientifiques de niveau recherche, publiés ou non, émanant des établissements d'enseignement et de recherche français ou étrangers, des laboratoires publics ou privés. 


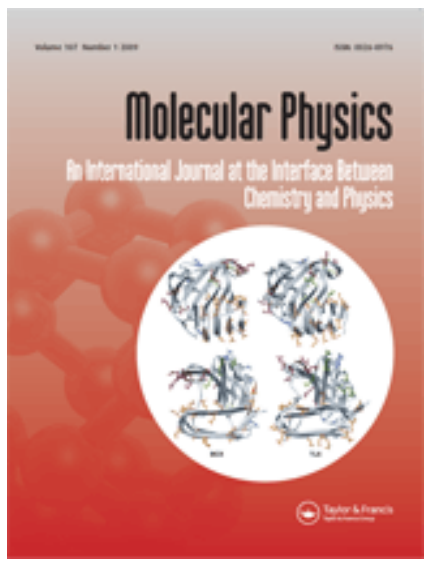

\section{Fluid of hard spheres with dipolar-like patch interaction and effect of adding an isotropic adhesion}

\begin{tabular}{|c|c|}
\hline Journal: & Molecular Physics \\
\hline Manuscript ID: & TMPH-2010-0306 \\
\hline Manuscript Type: & $\begin{array}{l}\text { Special Issue Paper -EIGHTH LIBLICE CONFERENCE on the } \\
\text { Statistical Mechanics of Liquids }\end{array}$ \\
\hline $\begin{array}{r}\text { Date Submitted by the } \\
\text { Author: }\end{array}$ & 26-Jul-2010 \\
\hline Complete List of Authors: & $\begin{array}{l}\text { Gazzillo, Domenico; Università Ca' Foscari, Dipartimento di Chimica } \\
\text { Fisica }\end{array}$ \\
\hline Keywords: & $\begin{array}{l}\text { anisotropic surface interactions, hard spheres with dipolar-like } \\
\text { patch, molecular Ornstein-Zernike integral equation, pair } \\
\text { correlation function, local orientational order }\end{array}$ \\
\hline
\end{tabular}

\section{SCHOLARONE Manuscripts}


Molecular Physics

Vol. 00, No. 00, Month 200x, 1-17

\title{
RESEARCH ARTICLE
}

\section{Fluid of hard spheres with dipolar-like patch interaction and effect of adding an isotropic adhesion.}

\author{
Domenico Gazzillo * \\ Dipartimento di Chimica Fisica, Università Ca' Foscari, \\ S. Marta Dorsoduro 2137, I-30123 Venezia, Italy \\ (Received 00 Month 200x; final version received 00 Month 200x)
}

\begin{abstract}
We compare two fluid models of spherical molecules with anisotropic, purely surface interactions. Both models admit an analytical solution of the molecular Ornstein-Zernike integral equation, within the Percus-Yevick approximation plus orientational linearization. In the first model, the molecular surface corresponds to a unique nonuniform patch, with a potential obtained by truncating a long-ranged dipolar interaction exactly at the contact distance between two hard sphere particles. In the second model, a further isotropic adhesion is added to the intermolecular potential. The study is focused on the local orientational ordering. Differences and similarities with respect to hard spheres with full long-ranged dipolar forces are analysed in detail. The effect of the competition between anisotropic patch interaction and isotropic adhesion is investigated through the pair correlation function as well as via two novel anisotropic order parameters.
\end{abstract}

Keywords: anisotropic surface interactions; hard spheres with dipolar-like patch; molecular Ornstein-Zernike integral equation; pair correlation function; local orientational order

\section{Introduction}

In the study of fluids with molecules interacting via anisotropic forces an important role is played by models which try to represent short-ranged directional interactions in a simple way. In many physical systems, interesting from both the theoretical and technological point of view (such as globular proteins, colloids, micelles, biomolecules, and nanoparticles able to self-assemble and build up novel materials), molecular interactions often act only when two particles are very close, or even only when their surfaces come into contact with each other.

In order to describe molecules with nonuniform surface interactions, a number of "patchy models" have been proposed and investigated. The simplest ones decorate the molecular surface with "sticky points" [1-7] or "sticky patches" [8-10]. Among the patchy potentials, the Kern-Frenkel model, originally due to Jackson et al. [8], has received a large renewed interest [11-13]. Its "uniform circular patches" have a well-defined circular boundary, are always attractive and characterized by an uniform strength of adhesion, which does not depend on the contact point within the patch.

In this paper we want to compare predictions on the local orientational ordering of two recent fluid models [14-16], whose molecules interact via anisotropic surface forces having dipolar-like symmetry. For both models, in which the entire molecular surface may be regarded as one nonuniform patch, the molecular Ornstein-Zernike

\footnotetext{
* Email: gazzillo@unive.it

ISSN: 0040-5167 print/ISSN 1754-2278 online (C) 200x Taylor \& Francis DOI: $10.1080 / 0040516 Y Y x x x x x x x x$ http://www.informaworld.com
} 
(OZ) integral equation

$$
h(1,2)=c(1,2)+\rho \int d \mathbf{r}_{3}\langle c(1,3) h(3,2)\rangle_{\mathbf{u}_{3}}
$$

was solved analytically, within the Percus-Yevick approximation plus orientational linearization (PY-OL closure) [14-16]. Here, $\rho$ is the number density, $h(1,2)$ and $c(1,2)$ are the total and direct correlation functions, respectively [17]. The symbol $i \equiv\left(\mathbf{r}_{i}, \mathbf{u}_{i}\right.$ ) (with $\left.i=1,2\right)$ includes, in addition to the position $\mathbf{r}_{i}$, also the orientation of molecule $i$, which can be specified - in all cases with axial symmetry - by a unit vector $\mathbf{u}_{i}$. Thus, the angular brackets with subscript $\mathbf{u}_{3}$ denote an average over all orientations of particle 3, i.e. $\langle\cdots\rangle_{\mathbf{u}_{3}}=(4 \pi)^{-1} \int d \mathbf{u}_{3} \cdots$.

Due to the dipolar-like symmetry of our surface interaction potentials, which will be specified in a while, the angular dependence of all correlation functions is expressed through the same set of angular functions $\{1, \Delta, D\}$ utilized by Wertheim for dipolar hard spheres (DHSs) [18]:

$$
\Delta(1,2)=\mathbf{u}_{1} \cdot \mathbf{u}_{2}, \quad D(1,2)=3\left(\mathbf{u}_{1} \cdot \hat{\mathbf{r}}\right)\left(\mathbf{u}_{2} \cdot \hat{\mathbf{r}}\right)-\Delta(1,2),
$$

where $\hat{\mathbf{r}}$ coincides with $\widehat{\mathbf{r}}_{12}=\mathbf{r}_{12} / r$, while $\mathbf{r}_{12}=\mathbf{r}_{2}-\mathbf{r}_{1}$ and $r=\left|\mathbf{r}_{12}\right|$.

Orientational linearization (OL) then means that, after expanding the correlation functions in power series of the basis functions $\{1, \Delta, D\}$, one neglects all terms after the three linear ones, i.e.

$$
c_{\mathrm{OL}}(1,2)=c_{0}(r)+c_{\Delta}(r) \Delta(1,2)+c_{D}(r) D(1,2),
$$

and similarly for $h_{\mathrm{OL}}(1,2)$.

In the DHS model, each particle is depicted as a hard sphere (HS) of diameter $\sigma$ with an embedded point dipole of strenght $\mu$ at its center. Because of the longranged nature of the dipole-dipole potential, any two molecules of this fluid can interact directly even when they are relatively far apart.

The two fluid models considered in the present paper stem, in some sense, from the DHS one; nevertheless, they strongly differ from it. In fact, their intermolecular potentials have not at all long-ranged dipolar tail, but represent forces acting only when two particles are at contact.

In the isotropic case, the first model with uniform "surface adhesion" was introduced by Baxter $[19,20]$. The corresponding "sticky hard sphere" (SHS) potential contains a HS repulsion plus a spherically symmetric attraction, described by a square-well which becomes infinitely deep and narrow, according to an appropriate limiting procedure (Baxter's sticky limit).

In the simplest of our anisotropic models the contact interaction is obtained by truncating the DHS potential exactly at $r=\sigma$. In Ref. [16] this model was referred to as dipolar sticky hard spheres, and denoted by the DSHS acronym. Since the same name was already used in the past by other authors [21, 22] for a different model (i.e. SHS molecules with an embedded point dipole, having full long-ranged dipolar tail), in the present paper we prefer to avoid any possible confusion by introducing the name of hard spheres with dipolar-like surface interaction, or simply HS particles with dipolar-like patch (DP model).

The "surface dipolar" interaction is anisotropic, can be both positive and negative (depending on the mutual orientation of the particles), and has the same angular 
dependence as the full DHS potential. Such an infinitely narrow dipolar potential may be regarded as an acceptable idealization for colloidal or protein suspensions, where the interaction range may be much shorter than the macroparticle size. A strong screening with a very small Debye length $\kappa_{\mathrm{D}}^{-1}$, due to a sufficiently dense ionic atmosphere in the solution, could cause all electrostatic forces to vanish close to the molecular surface, and become truncated exactly at contact in the idealized sticky limit.

The second model - already studied in Refs. $[14,15]$ - is composed of HSs with an anisotropic surface interaction, which is purely attractive and comes out from the sum of a uniform surface adhesion (isotropic background) plus an appropriate dipolar sticky correction. In the present paper, we propose a reinterpretation of this second model: in fact, one can regard it as the result of adding an isotropic adhesion to the DP particles of the first model. Since a term has been added to the DP potential, this second model will be referred to as DP+ fluid.

The aim of the present paper is twofold. First, we want to complement Ref. [16], by getting more insight into the orientational structure of the DP model, clear up some details and correct a mistake of that paper. Second, we will compare the local orientational structures predicted by the DP and DP + models, in order to investigate the effect of the isotropic adhesion, in competition with the dipolar-like surface term.

\section{Hard spheres with dipolar-like patch}

For the DHS model, whose dipole-dipole potential is $\phi_{\mathrm{dd}}(1,2)=-\left(\mu^{2} / r^{3}\right) D(1,2)$, Wertheim [18] found an analytic solution of the OZ equation within the mean spherical approximation (MSA), i.e. assuming that

$$
\left\{\begin{array}{l}
c_{0}(r)=0 \\
c_{\Delta}(r)=0 \\
c_{D}(r)=\beta \mu^{2} / r^{3}
\end{array} \quad \text { when } r \geq \sigma\right.
$$

where $\beta=\left(k_{B} T\right)^{-1}\left(k_{B}\right.$ being the Boltzmann constant, $T$ the absolute temperature). Clearly, the dipole-dipole interaction may be both attractive (for molecular configurations with $D>0$ ) and repulsive (when $D<0$ ).

To define both our DP model and the PY-OL closure, it is sufficient to specify the approximation to the direct correlation function outside the core [16], i.e.

$$
\begin{gathered}
\left\{\begin{array}{l}
c_{0}(r)=0 \\
c_{\Delta}(r)=0 \\
c_{D}(r)=\Lambda_{D} \sigma \delta(r-\sigma)
\end{array} \quad \text { when } r \geq \sigma,\right. \\
\Lambda_{D}=t_{\mathrm{d}} y_{0}(\sigma), \quad t_{\mathrm{d}}=\left(\mu^{*}\right)^{2} \equiv \frac{\beta \mu^{2}}{\sigma^{3}}, \quad y_{0}(\sigma)=\frac{1+\frac{1}{2} \eta}{(1-\eta)^{2}},
\end{gathered}
$$

where the reduced dipolar strength $t_{\mathrm{d}}=\left(\mu^{*}\right)^{2}$ denotes the ratio of the dipolar interaction energy at contact to the thermal energy, while $y_{0}(\sigma)$ is the PY contact value of the HS cavity function, $y_{0}(r)=g_{0}(r) \exp \left[\beta \phi_{\mathrm{HS}}(r)\right]$, and $\eta=(\pi / 6) \rho \sigma^{3}$ is 
the volume fraction. The Dirac delta function $\delta(r-\sigma)$ ensures that the dipolar-like interaction acts only at contact.

Note that, whereas the MSA employed by Wertheim for the DHS model is density-independent, the actual PY-OL closure is density-dependent through the factor $y_{0}(\sigma)$, which is proportional to the probability of finding a particle touching any given particle. As $\eta \rightarrow 0, c(1,2)$ reduces to the Mayer function

$$
f_{\mathrm{DP}}(1,2)=f_{0}(r)+t_{\mathrm{d}} D(1,2) \sigma \delta(r-\sigma),
$$

which can also be regarded as a way of defining the model $\left(f_{0}(r)\right.$ coincides with $f_{\mathrm{HS}}(r)=\Theta(r-\sigma)-1$, with $\left.\Theta(x<0)=0, \Theta(x>0)=1\right)$.

The fully analytic PY-OL solution found in Ref. [16] allows to calculate, in particular, the pair distribution function $g(1,2)=1+h(1,2)$ :

$$
g(1,2)=1+h_{0}(r)+h_{\Delta}(r) \Delta(1,2)+h_{D}(r) D(1,2) .
$$

It results that

$$
\left\{\begin{array}{l}
h_{0}(r)=h_{\mathrm{HS}}(r ; \eta) \\
h_{\Delta}(r)=h_{\Delta, \mathrm{reg}}(r) \\
h_{D}(r)=h_{D, \operatorname{reg}}(r)+\Lambda_{D} \sigma \delta(r-\sigma),
\end{array}\right.
$$

with

$$
\begin{gathered}
h_{D, \mathrm{reg}}(r)=h_{D, \mathrm{reg}}^{0}(r)+\frac{3}{r^{3}}\left[\left(K-\Lambda_{D}\right) \sigma^{3}-\int_{\sigma}^{r} h_{D, \mathrm{reg}}^{0}(x) x^{2} d x\right] \quad \text { for } r \geq \sigma \\
\left\{\begin{array}{l}
h_{\Delta, \text { reg }}(r)=2 K\left[h_{\mathrm{SHS}, \mathrm{reg}}\left(r ; \eta_{2}, \Lambda\right)-h_{\mathrm{SHS}, \mathrm{reg}}\left(r ; \eta_{1}, \Lambda\right)\right] \\
h_{D, \mathrm{reg}}^{0}(r)=2 K\left[h_{\mathrm{SHS}, \mathrm{reg}}\left(r ; \eta_{2}, \Lambda\right)+\frac{1}{2} h_{\mathrm{SHS}, \mathrm{reg}}\left(r ; \eta_{1}, \Lambda\right)\right]
\end{array}\right.
\end{gathered}
$$

where $\Lambda \equiv \Lambda_{D} /(3 K)$, the subscript "SHS" refers to an isotropic sticky HS fluid, "reg" denotes the regular part (with no $\delta$-singularity, and - at most - some step discontinuities), while the singular $\delta$-term corresponds to the surface interaction. Moreover,

$$
\left\{\begin{array}{l}
\eta_{1}=-K \eta \\
\eta_{2}=2 K \eta
\end{array}\right.
$$

and the parameter $K$ is explicitly given by

$$
K=\frac{\Lambda_{D}}{\frac{1}{3}\left(1+\xi_{\mathrm{d}}+\sqrt[3]{\mathcal{A}-\sqrt{\Delta}}+\sqrt[3]{\mathcal{A}+\sqrt{\Delta}}\right)}
$$

with $\xi_{\mathrm{d}}=\Lambda_{D} \eta$, and $\Delta \equiv \mathcal{A}^{2}-\mathcal{B}^{3}>0$,

$$
\mathcal{A}=1+\frac{15}{8} \xi_{\mathrm{d}}+\frac{195}{8} \xi_{\mathrm{d}}^{2}+10 \xi_{\mathrm{d}}^{3}, \quad \mathcal{B}=1+\frac{5}{4} \xi_{\mathrm{d}}+7 \xi_{\mathrm{d}}^{2},
$$

Eqs. (13) and (14) replace their counterparts of Ref. [16], which were partly incorrect. 


\section{Molecular Physics}

Molecular Physics

\section{Adding an isotropic adhesion}

In Refs. $[14,15]$ the second model was defined, in terms of Mayer function, as

$$
f_{\mathrm{DP}+}(1,2)=f_{\mathrm{HS}}(r)+[t+(\alpha t) D(1,2)] \sigma \delta(r-\sigma),
$$

which may be considered as the sum of a reference and an excess part, according to the splitting

$$
\begin{gathered}
f_{\mathrm{DP}+}(1,2)=f_{0}(r)+f_{\mathrm{ex}}(1,2), \\
\left\{\begin{array}{l}
f_{0}(r)=f_{\mathrm{HS}}(r)+t \sigma \delta(r-\sigma) \equiv f_{\mathrm{SHS}}(r) \\
f_{\mathrm{ex}}(1,2)=(\alpha t) \quad \sigma \delta(r-\sigma) D(1,2) .
\end{array}\right.
\end{gathered}
$$

In this way, the reference system is a SHS fluid with isotropic adhesion, while $f_{\text {ex }}(1,2)$ denotes the dipolar-like patch correction.

The PY-OL closure reads as

$$
\left\{\begin{array}{l}
c_{0}(r)=\Lambda_{0} \sigma \delta(r-\sigma) \\
c_{\Delta}(r)=\Lambda_{\Delta} \sigma \delta(r-\sigma) \\
c_{D}(r)=\Lambda_{D} \sigma \delta(r-\sigma)
\end{array} \quad \text { for } r \geq \sigma\right.
$$

with

$$
\left\{\begin{array}{l}
\Lambda_{0}=t y_{0}(\sigma) \\
\Lambda_{\Delta}=t y_{\Delta}(\sigma) \\
\Lambda_{D}=t y_{D}(\sigma)+(\alpha t) y_{0}(\sigma)
\end{array}\right.
$$

where $y_{0}(\sigma), y_{\Delta}(\sigma)$ and $y_{D}(\sigma)$ denote the coefficients of the PY cavity function at contact $[14,15]$.

The PY-OL solution leads to

$$
\left\{\begin{array}{c}
h_{0}(r)=h_{0, \mathrm{reg}}(r)+\Lambda_{0} \sigma \delta(r-\sigma) \\
h_{\Delta}(r)=h_{\Delta, \mathrm{reg}}(r)+\Lambda_{\Delta} \sigma \delta(r-\sigma) \\
h_{D}(r)=h_{D, \mathrm{reg}}(r)+\Lambda_{D} \sigma \delta(r-\sigma)
\end{array}\right.
$$

where $h_{D, \text { reg }}(r)$ is again given by Eq. (10), while

$$
\left\{\begin{array}{l}
h_{\Delta, \text { reg }}(r)=2 K\left[h_{\mathrm{SHS}, \text { reg }}\left(r ; \eta_{2}, \Lambda_{2}\right)-h_{\mathrm{SHS}, \text { reg }}\left(r ; \eta_{1}, \Lambda_{1}\right)\right] \\
h_{D, \text { reg }}^{0}(r)=2 K\left[h_{\mathrm{SHS}, \mathrm{reg}}\left(r ; \eta_{2}, \Lambda_{2}\right)+\frac{1}{2} h_{\mathrm{SHS}, \mathrm{reg}}\left(r ; \eta_{1}, \Lambda_{1}\right)\right]
\end{array}\right.
$$

(for the definition of $\Lambda_{1}$ and $\Lambda_{2}$ we refer to Ref. [14]). The parameter $K$ differs from that given by Eq. (13), and its value must be calculated by solving one equation numerically.

In the present paper, we want to compare the DP+ and DP models. To this aim, it is convenient to imagine a different splitting for $f_{\mathrm{DP}+}(1,2)$, i.e.

$$
\left\{\begin{array}{l}
f_{0}(1,2)=f_{\mathrm{HS}}(r)+t_{\mathrm{d}} \sigma \delta(r-\sigma) D(1,2) \equiv f_{\mathrm{DP}}(1,2) \\
f_{\mathrm{ex}}(r)=t \sigma \delta(r-\sigma)
\end{array}\right.
$$


after equating

$$
t_{\mathrm{d}} \equiv \alpha t
$$

The parameter $t_{\mathrm{d}}$ defines the strength of the dipolar-like patch interaction, $t$ controls the isotropic surface attraction, while $\alpha=t_{\mathrm{d}} / t$ measures the anisotropy degree of the resulting potential.

\section{The pair correlation function}

The knowledge of the pair correlation function $g(1,2)$ allows to get all structural and thermodynamic properties of the fluid. In particular, it is possibile to investigate both the structure and the orientational order around any generic particle 1, chosen as reference molecule. In fact, using Eq. (8), one can determine $g_{\mathrm{PY}-\mathrm{OL}}(1,2)$ for any position and orientation of a test particle 2 .

In general, $g_{\mathrm{PY}-\mathrm{OL}}(1,2)$ is a function of the variables $\left(r, \hat{\mathbf{r}}, \mathbf{u}_{1}, \mathbf{u}_{2}\right)$ : its dependence on $r$ is specified by the coefficients $\left\{h_{0}, h_{\Delta}, h_{D}\right\}$, while the angular basis functions, $\Delta(1,2)$ and $D(1,2)$ include the unit vectors $\hat{\mathbf{r}}, \mathbf{u}_{1}, \mathbf{u}_{2}$. Thus, after calculating $\left\{h_{0}, h_{\Delta}, h_{D}\right\}$ as functions of $r$, one has only to evaluate $\Delta$ and $D$ for the configurations of interest.

As polar axis (also Cartesian $z$ axis) we choose the straight half-line originating from the centre $\mathbf{r}_{1}$ of molecule 1 and having orientation $\mathbf{u}_{1}$ (see Figure 1). Then, as polar plane for evaluating azimuthal angles $\varphi$, we adopt that defined by the unit vectors $\left(\mathbf{u}_{1}, \hat{\mathbf{r}}\right)$.

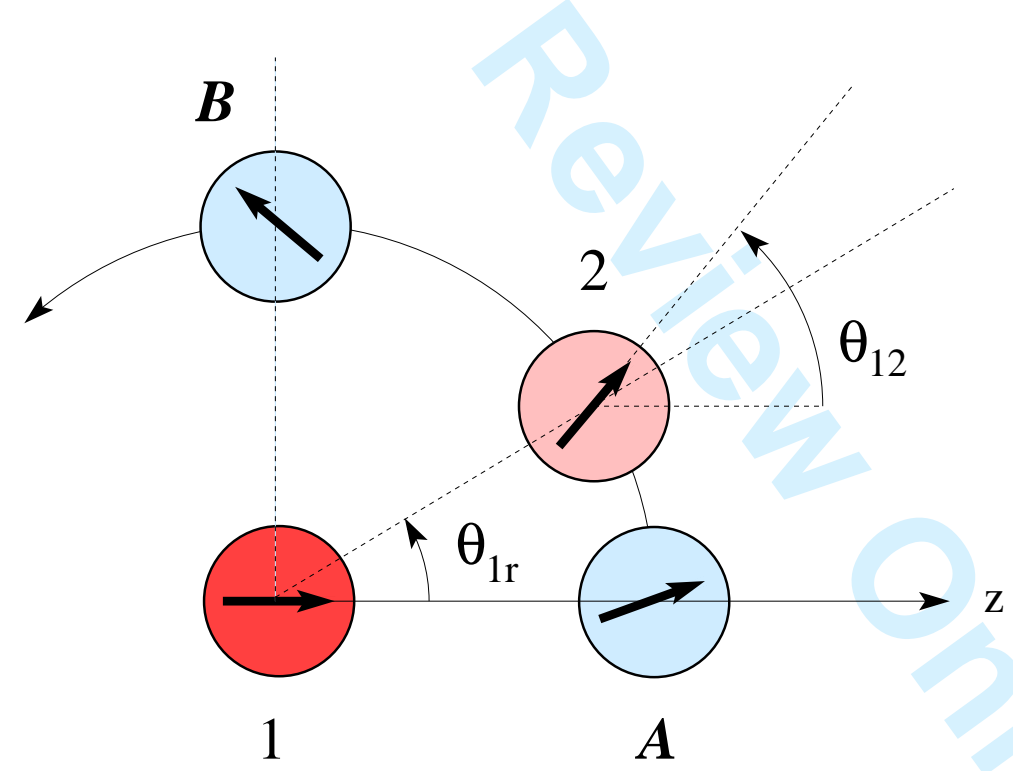

Figure 1. A test particle 2 explores the region around a generic reference particle 1 . At fixed $r$, the angle $\theta_{1 r}$ increases from $0^{\circ}$ to $180^{\circ}$. Then, for each fixed value of $\theta_{1 r}, \theta_{12}$ increases from $0^{\circ}$ to $360^{\circ}$, as the test particle rotates counterclockwise.

For equilibrium configurations, symmetry requires that $\mathbf{u}_{2}$ must be coplanar with the vectors $\left(\mathbf{u}_{1}, \hat{\mathbf{r}}\right)$, i.e. $\varphi_{1}=\varphi_{2}=\varphi_{r}=0$ [16]. As a consequence, $\Delta$ and $D$ reduce to

$$
\begin{gathered}
\Delta=\cos \theta_{12} \\
D=3 \cos \theta_{1 r} \cos \left(\theta_{12}-\theta_{1 r}\right)-\cos \theta_{12},
\end{gathered}
$$


where $\theta_{1 r}$ is the angle between $\mathbf{u}_{1}$ and $\hat{\mathbf{r}}$, while $\theta_{12}$ characterizes the direction of $\mathbf{u}_{2}$ (with $-\pi \leq \theta_{12}<\pi$, or $0 \leq \theta_{12}<2 \pi$ ).

Now, we can simply write $g_{\mathrm{PY}-\mathrm{OL}}\left(r, \theta_{1 r}, \theta_{12}\right)$, and calculate it as a function of $r$, for several values of $\theta_{1 r}$ and $\theta_{12}$. Let us imagine to fix $r$ and vary $\theta_{1 r}$ from $0^{\circ}$ to $180^{\circ}$. Then, for each fixed $\theta_{1 r}$, we rotate $\mathbf{u}_{2}$ counterclockwise by increasing $\theta_{12}$ from $0^{\circ}$ to $360^{\circ}$. At a given position $\left(r, \theta_{1 r}\right)$, the most probable orientation of particle 2 is identified by the corresponding relative maximum of $g$.

In particular, for all points belonging to the polar axis, such as molecule $A$ in Figure 1, one has $\theta_{1 r}=0, D_{A}=2 \cos \theta_{12}$, and thus

$$
g_{A}\left(r, \theta_{12}\right)=g_{0}(r)+\left[h_{\Delta}(r)+2 h_{D}(r)\right] \cos \theta_{12}
$$

where $g_{0}(r) \equiv 1+h_{0}(r)$. On the other hand, on the axis perpendicular (see molecule $B$ of the same Figure) one finds $\theta_{1 r}=\pi / 2$ and $D_{B}=-\cos \theta_{12}$, which leads to

$$
g_{B}\left(r, \theta_{12}\right)=g_{0}(r)+\left[h_{\Delta}(r)-h_{D}(r)\right] \cos \theta_{12} .
$$

\section{DP model}

When defining the DP model, no range was specified for $t_{\mathrm{d}}$. Reasonably, only rather small values are expected to be allowed, since the OL-approximation should break down at stronger dipolar strength. We have however adopted a pragmatic point of view. For each considered density $\rho^{*} \equiv \rho \sigma^{3}$, an approximate upper bound $\left(t_{\mathrm{d}}\right)_{\max }$ has been determined numerically, by searching for the point at which $g_{\mathrm{PY}-\mathrm{OL}}$ starts to exhibit some pathological negative values (as occurs, within the PY approximation, even for the HS fluid at high density).

It results that: if $\rho^{*}=0.1$, then $\left(t_{\mathrm{d}}\right)_{\max } \approx 1.4$, whereas at $\rho^{*}=0.8$ the value of $t_{\mathrm{d}}$ cannot exceed $\approx 0.18$. In the density range $0.1 \div 0.8$, the product $\rho^{*}\left(t_{\mathrm{d}}\right)_{\max }$ always remains in the interval $0.14 \div 0.19$ (reasonably, the most important variable should be $\left.\xi_{\mathrm{d}}=t_{\mathrm{d}} \eta y(\sigma)\right)$.

Figures $2 \mathrm{a})$ and $2 \mathrm{~b})$ show $g_{A}\left(r, \theta_{12}\right)$ and $g_{B}\left(r, \theta_{12}\right)$, respectively, when $\eta=0.4$ $\left(\rho^{*}=0.764\right)$ and $t_{\mathrm{d}}=0.2$, for three significant orientations of the test particle 2 : $\theta_{12}=0^{\circ}$ (parallel), $\theta_{12}=90^{\circ}$ (orthogonal), and $\theta_{12}=180^{\circ}$ (antiparallel).

Recalling - from Eq. (9) - that in the DP model $h_{D}(r)$ includes a surface $\delta$-term, one realizes that - apart from the orthogonal configuration - both $g_{A}$ and $g_{B}$ contain a singular contribution, i.e. $2 \Lambda_{D} \cos \theta_{12} \sigma \delta(r-\sigma)$ and $-\Lambda_{D} \cos \theta_{12} \sigma \delta(r-\sigma)$, respectively.

Perhaps, in Ref. [16] the role and importance of these singular surface contributions was not sufficiently emphasized. In fact, for all configurations with $r>\sigma$ these terms can be neglected, and the structure is fully determined only by the regular part of $g$. On the contrary, for all configurations with direct contact $(r=\sigma)$ the $\delta$-terms cannot be omitted, but must be properly taken into account. Since these direct contact cases were not discussed in detail and unambiguously in Ref. [16], we will make up for this shortcoming here.

Due to the nature of the Dirac $\delta$ which is not a function in the usual sense, the presence of the singular surface terms is not apparent in Figure 2, where they have not been depicted. One could represent them symbolically as spikes, by approximating the Dirac $\delta$-distribution with a "rectangular" function $\delta_{w}(r) \geq 0$, centered at $\sigma$, and having an extremely narrow width $w$ with a very large height $w^{-1}$. The replacement $\delta \rightarrow \delta_{w}$ will also be useful in the following, in order to get a simpler picture for the interaction of molecules 1 and 2 when they are at contact. Physically, it introduces a "contact surface layer" of width $w$. We will then imagine to 

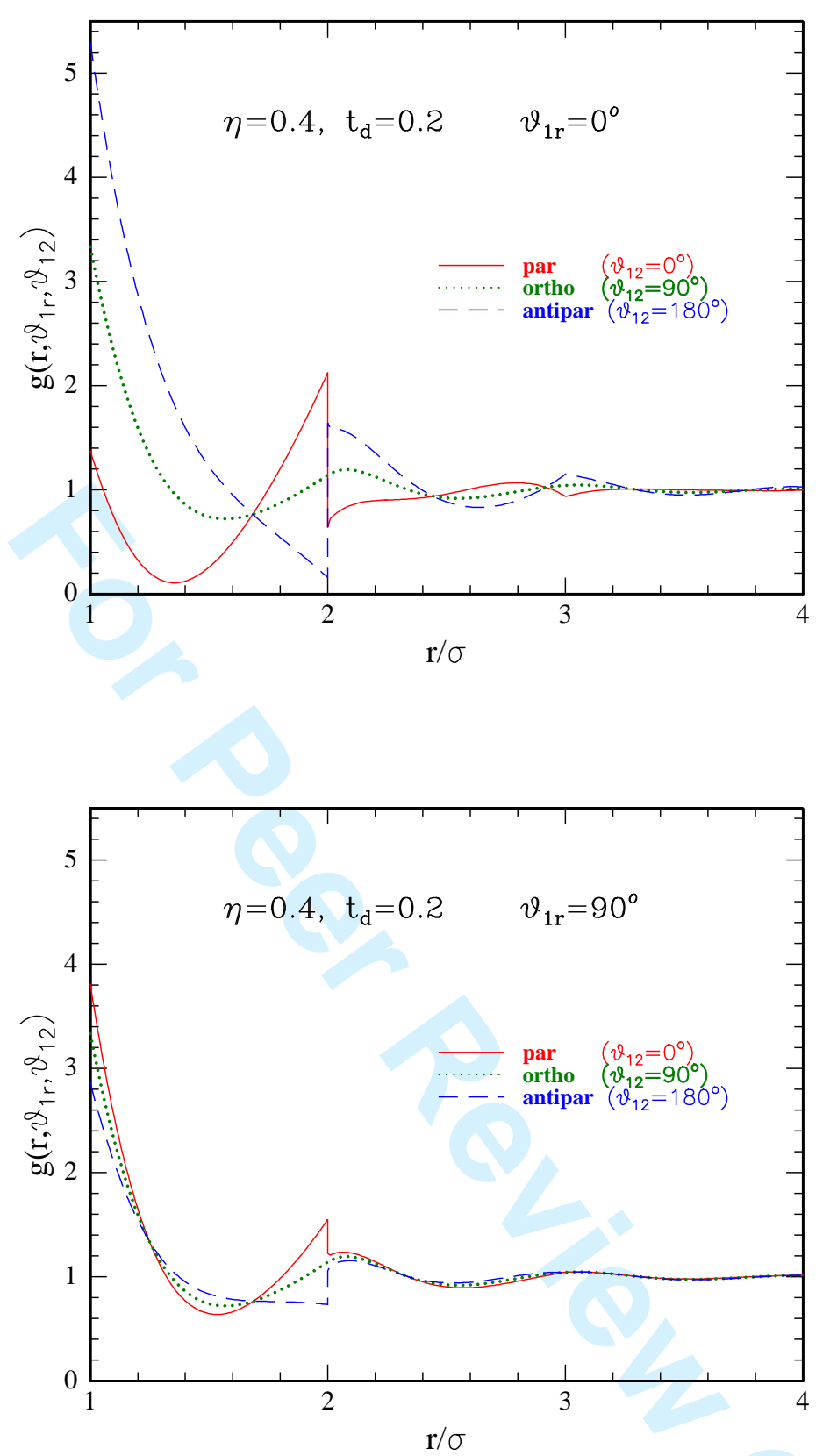

Figure 2. Fluid of hard spheres only with dipolar-like patch interaction. Upper panel a): regular part of $g_{A}\left(r, \theta_{12}\right) \equiv g\left(r, \theta_{1 r}=0^{\circ}, \theta_{12}\right)$, at $\eta=0.4$ and $t_{\mathrm{d}}=0.2$, for three representative orientations: $\theta_{12}=$ $0^{\circ}, 90^{\circ}$, and $180^{\circ}$ (parallel, orthogonal, and antiparallel configurations). Lower panel b): regular part of $g_{B}\left(r, \theta_{12}\right) \equiv g\left(r, \theta_{1 r}=90^{\circ}, \theta_{12}\right)$, for the same cases as in panel a).

take the limit $w \rightarrow 0$ only at the end of our reasoning.

\subsection{Direct interactions}

When $\rho^{*} \rightarrow 0$ and molecules 1 and 2 are alone, their interactions can occur only at contact. It is important to realize that, in spite of the difference in strength, these impulsive surface forces favour exactly the same mutual orientations as the long-ranged dipolar interactions of the DHS model do at contact.

In fact, replacing $\delta$ with $\delta_{w}$, the surface terms of $g_{A}$ and $g_{B}$ become, respectively, 
$2 t_{\mathrm{d}} \cos \theta_{12} \sigma \delta_{w}(r)$ and $-t_{\mathrm{d}} \cos \theta_{12} \sigma \delta_{w}(r)$, which differ from zero only in the narrow contact layer with width $w$. When molecule $A$ of Figure 1 moves along the polar axis and touches molecule 1 , the most probable angular configuration - which always corresponds to a relative maximum of $g$ - is the parallel one, since the contact value $g_{A}\left(\sigma^{+}\right)=2 t_{\mathrm{d}} \cos \theta_{12} \sigma / w$ attains its largest positive value, $2 t_{\mathrm{d}} \sigma / w$, when $\theta_{12}=0^{\circ}$. On the other hand, for molecules moving along the axis $B$ orthogonal to the polar one, the most probable contact orientation is the antiparallel one (the relative maximum of $g_{B}\left(\sigma^{+}\right)=-t_{\mathrm{d}} \cos \theta_{12} \sigma / w$ is $t_{\mathrm{d}} \sigma / w$, corresponding to $\theta_{12}=180^{\circ}$ ). Note that $g_{A}(\sigma)>g_{B}(\sigma)$, and from the study of all other configurations, when molecules 1 and 2 are alone and at contact, it comes out that the "head-to-tail"arrangement of the two dipoles is the most probable, just as in the DHS model.

On the contrary, when $\rho^{*} \neq 0$ the behaviour of our DP model strongly differs from the DHS one. The main difference consists in the fact that, since the range of the dipolar-like patch potential has null width, the interaction between molecules 1 and 2 with $r>\sigma$ can only be "indirect", i.e. mediated by a chain of one or more intermediate particles ("mediators" of interaction), each in direct contact with the neighbouring ones. In order to get more insight into the behaviour of $g$, it is thus convenient to focus on these indirect interactions.

\subsection{Indirect interactions}

In general, the density expansion of $g(1,2)$ reads as

$$
g(1,2)=[1+f(1,2)]\left[1+\sum_{m=1}^{\infty} y^{(m)}(1,2) \rho^{m}\right] .
$$

The coefficient $y^{(m)}(1,2)$ of $\rho^{m}$ can be expressed by multiple integrals, which refer to all possible interactions of a cluster of $n=2+m$ molecules, with the "root particles" 1 and 2 having fixed positions and orientations, whereas the $m$ "field particles" assume all possible positions and orientations.

In the DP model, due to the peculiar form of the potential, the only configurations yielding a non-vanishing contribution to $y^{(m)}(1,2)$ are those where each of the $n$ particles has at least one contact with another particle of the cluster. Consequently, $y^{(1)}(1,2) \rho$ represents the contribution of all possible "trimers" $1-3-2$, while $y^{(2)}(1,2) \rho^{2}$ that of "tetramers", and so on (with the superscript denoting the number of "mediators" or "field" particles). The values of $g(1,2)$ are determined by the contributions of all dimers, trimers, tetramers, and higher-order aggregates.

However, since the maximum size of a cluster cannot exceed the length of its "linear chain" configuration, the dimers can give a non-zero contribution only at contact $(r=\sigma)$, while the trimer term $y^{(1)}(1,2)$ differs from zero only in the interval $\left(\sigma, 2 \sigma^{-}\right)$, and in general the integral $y^{(n-2)}(1,2)$ corresponding to " $n$-mers" vanishes for $r \geq(n-1) \sigma$. As a consequence, in the interval $\left(\sigma, 2 \sigma^{-}\right)$the leading term is that of trimers; in $\left(2 \sigma, 3 \sigma^{-}\right)$the leading term is due to tetramers; and so on.

Thus, since we are mainly interested in the first layer $\left(\sigma, 2 \sigma^{-}\right)$, calculating $y^{(1)}(1,2)$ could be enough for a qualitative or semiquantitative analysis. We can write

$$
g(1,2)=[1+f(1,2)]\left[1+y^{(1)}(1,2) \rho+\mathcal{O}\left(\rho^{2}\right)\right]
$$




\section{Molecular Physics}

where

$$
y^{(1)}(1,2)=\int d \mathbf{r}_{3}\langle f(1,3) f(3,2)\rangle_{\mathbf{u}_{3}}
$$

is the "one-mediator" term (higher order mediators are not essential at this level). Adding the OL approximation then consists in neglecting, in Eqs. (28)-(29), all terms non-linear with respect to the angular basis functions $\{\Delta, D\}$.

The contribution of "trimers" in the interval $(\sigma, 2 \sigma)$ leads to

$$
\begin{gathered}
g_{A, \mathrm{reg}}\left(x, \theta_{12}\right)-g_{\mathrm{HS}}(x)=\frac{t_{\mathrm{d}}^{2} \eta}{2 x}\left(9 x^{4}-30 x^{2}+8\right) \cos \theta_{12}+\mathcal{O}\left(\eta^{2}\right), \\
g_{B, \mathrm{reg}}\left(x, \theta_{12}\right)-g_{\mathrm{HS}}(x)=\frac{t_{\mathrm{d}}^{2} \eta}{4 x}\left(9 x^{4}-30 x^{2}+40\right) \cos \theta_{12}+\mathcal{O}\left(\eta^{2}\right),
\end{gathered}
$$

with $x \equiv r / \sigma$. When $1<x<2$, particles 1 and 2 do not touch each other, but the "mediator" particle 3 , in direct contact with both of them, gives rise to an indirect bonding.

The sign of the difference $g_{A, \text { reg }}-g_{\mathrm{HS}}$ changes at $x \approx \sqrt{3}$, as in Figure $2 \mathrm{a}$ ). In qualitative agreement with Figures $2 \mathrm{a}$ ) and $2 \mathrm{~b}$ ), one then finds:

$$
\begin{aligned}
& g_{A, \mathrm{reg}}\left(1^{+}, \theta_{12}\right)-g_{\mathrm{HS}}\left(1^{+}\right) \approx-(13 / 2) t_{\mathrm{d}}^{2} \eta \cos \theta_{12} \\
& g_{A, \mathrm{reg}}\left(2^{-}, \theta_{12}\right)-g_{\mathrm{HS}}\left(2^{-}\right) \approx 8 t_{\mathrm{d}}^{2} \eta \cos \theta_{12}
\end{aligned}
$$

and

$$
\begin{aligned}
& g_{B, \mathrm{reg}}\left(1^{+}, \theta_{12}\right)-g_{\mathrm{HS}}\left(1^{+}\right) \approx(19 / 4) t_{\mathrm{d}}^{2} \eta \cos \theta_{12} \\
& g_{B, \mathrm{reg}}\left(2^{-}, \theta_{12}\right)-g_{\mathrm{HS}}\left(2^{-}\right) \approx 8 t_{\mathrm{d}}^{2} \eta \cos \theta_{12},
\end{aligned}
$$

respectively. As a consequence, we get $g_{A \text {,reg }}^{\text {antipar }}>g_{A, \text { reg }}^{\text {par }}$ (but $g_{B \text {,reg }}^{\text {antipar }}<g_{B, \text { reg }}^{\text {par }}$ ) at contact $x=1^{+}$, whereas $g_{A, \text { reg }}^{\mathrm{par}}>g_{A, \text { reg }}^{\text {antipar }}\left(\right.$ and $g_{B, \text { reg }}^{\mathrm{par}}>g_{B, \text { reg }}^{\text {antipar }}$ ) at $x=2^{-}$.

However, at contact - in addition to the indirect contributions represented by the regular parts of $g$ - the direct, singular $\delta$-terms must also be taken into account. Within the $\delta_{w}$-approximation, the complete pair correlation functions in the contact surface layer of width $w$ become:

$$
\begin{aligned}
& g_{A}\left(1^{+}, \theta_{12}\right)-g_{\mathrm{HS}}\left(1^{+}\right) \approx\left[2 t_{\mathrm{d}} \sigma / w-(13 / 2) t_{\mathrm{d}}^{2} \eta\right] \cos \theta_{12} \\
& g_{B}\left(1^{+}, \theta_{12}\right)-g_{\mathrm{HS}}\left(1^{+}\right) \approx\left[-t_{\mathrm{d}} \sigma / w+(19 / 4) t_{\mathrm{d}}^{2} \eta\right] \cos \theta_{12}
\end{aligned}
$$

respectively. In both cases, due to the smallness of $w$, the singular term overcomes the regular one. In conclusion, we can state that, even at finite density, the most probable orientations of molecule 2 within the contact layer coincide with those of the long-ranged DHS model. On the contrary, moving outside the contact layer along the polar axis, antiparallel configurations are first preferred when $1<x \lesssim$ $\sqrt{3}$. Then, in the interval $\sqrt{3} \lesssim x<2$ the parallel orientation becomes again the most probable, as shown by the large peak at $x=2^{-}$in Figure 2a). Finally, for $x>$ 2 all curves exhibit some oscillations, with alternating preference for antiparallel and parallel configurations. The rapid decay of their amplitudes with increasing 
$r$ however means that, at least in the examined cases, the orientational order is purely local.

It is worth noting that, from a comparison between $g_{A}$ and $g_{B}$ results of Figures 2a) and $2 \mathrm{~b}$ ), it results that $g_{A} \gtrsim g_{B}$. Such a finding seems to signal a trend to form small portions of chains along the polar axis, just as in the DHS model. On the other hand, the alternance of preferred parallel and antiparallel orientations with increasing $r$ leads to conclude that truncating at contact the long-ranged dipolar interaction yields a local orientational structure really different from the DHS case. The sharp inversion of the most probable orientations in passing from inside to outside the surface layer of width $w$ is however a peculiar feature of our strongly idealized model. For a more realistic potential such a transition would probably be smooth.

\section{6. $\mathrm{DP}+$ model}

Figure 3 illustrates what happens to $g_{A \text {,reg }}\left(r, \theta_{12}\right)$ and $g_{B \text {,reg }}\left(r, \theta_{12}\right)$ when an isotropic adhesion is added to the molecular interaction potential. We have fixed $\eta=0.4$ with $t_{\mathrm{d}}=0.2$, giving several increasing values to $t$ : $0.01,0.1,0.2,0.4$, etc. Figure 3 refers only to $t=0.1$, since the other results are qualitatively similar.

A first, but not essential, remark is that in the DP + model even $g_{0}(r)$ exhibits a discontinuity at $r=2 \sigma$. The main result is, however, that adding an isotropic adhesion does not modify the relative position of the curves, with respect to Figure 2 , but can strongly reduce both $g_{A \text {,reg }}$ and $g_{B \text {,reg }}$, depending on the value of $t$ which represents the strength of the isotropic contribution. The physical reason is that the isotropic adhesion can now attach a molecule 2 at whatever surface point of molecule 1. Thus it competes with the dipolar-like surface interaction, destroying the local orientational order induced by the latter along the preferred direction of the polar axis.

It is worthwhile recalling, once again, that Figure 3 depicts only the regular part of $g$ related to indirect interactions, while the $\delta$-terms of the direct contact interactions have not been plotted, but are equally essential.

\section{Anisotropic indicators of orientational order}

The orientational ordering of these models can alternatively be investigated by taking into account both the regular and $\delta$-singular parts of $g(1,2)$ simultaneously. This second approach requires the evaluation of integrals with integrand functions involving $g(1,2)$.

As a measure of dipolar alignment, here we choose the value of $\mathbf{u}_{1} \cdot \mathbf{u}_{2}=\cos \theta_{12}$ averaged over a peculiar volume $V_{A B}$ connected to the reference particle $1[15,16]$. More precisely, let us start with the finite spherical region $S_{R}$ centered on molecule 1 and having radius $R$. Then, we consider the portion $V_{A B}$ of $S_{R}$ characterized by a specific solid angle, i.e. $\Omega_{A B}=\left\{\left(\theta_{1 r}, \varphi_{r}\right) \mid \theta_{A} \leq \theta_{1 r} \leq \theta_{B}, 0 \leq \varphi_{r} \leq 2 \pi\right\}$ (the polar axis being again defined by $\left.\mathbf{u}_{1}\right)$.

Figure 4 shows the three most important cases. The first one, with $\theta_{A}=0$ and $\theta_{B}=\pi$, yields the whole volume $V_{\text {sphere }}$ of the sphere $S_{R}$. The second region, $V_{\text {forward with }} \theta_{A}=0$ and $\theta_{B}=\pi / 3$, is conical and useful to investigate the "forward ordering" as seen by the particle 1 . Finally, taking $\theta_{A}=\pi / 3$ and $\theta_{B}=\pi / 2$ leads to a ring-shaped volume $V_{\text {lateral }}$, which allows to analyse the "lateral ordering." 

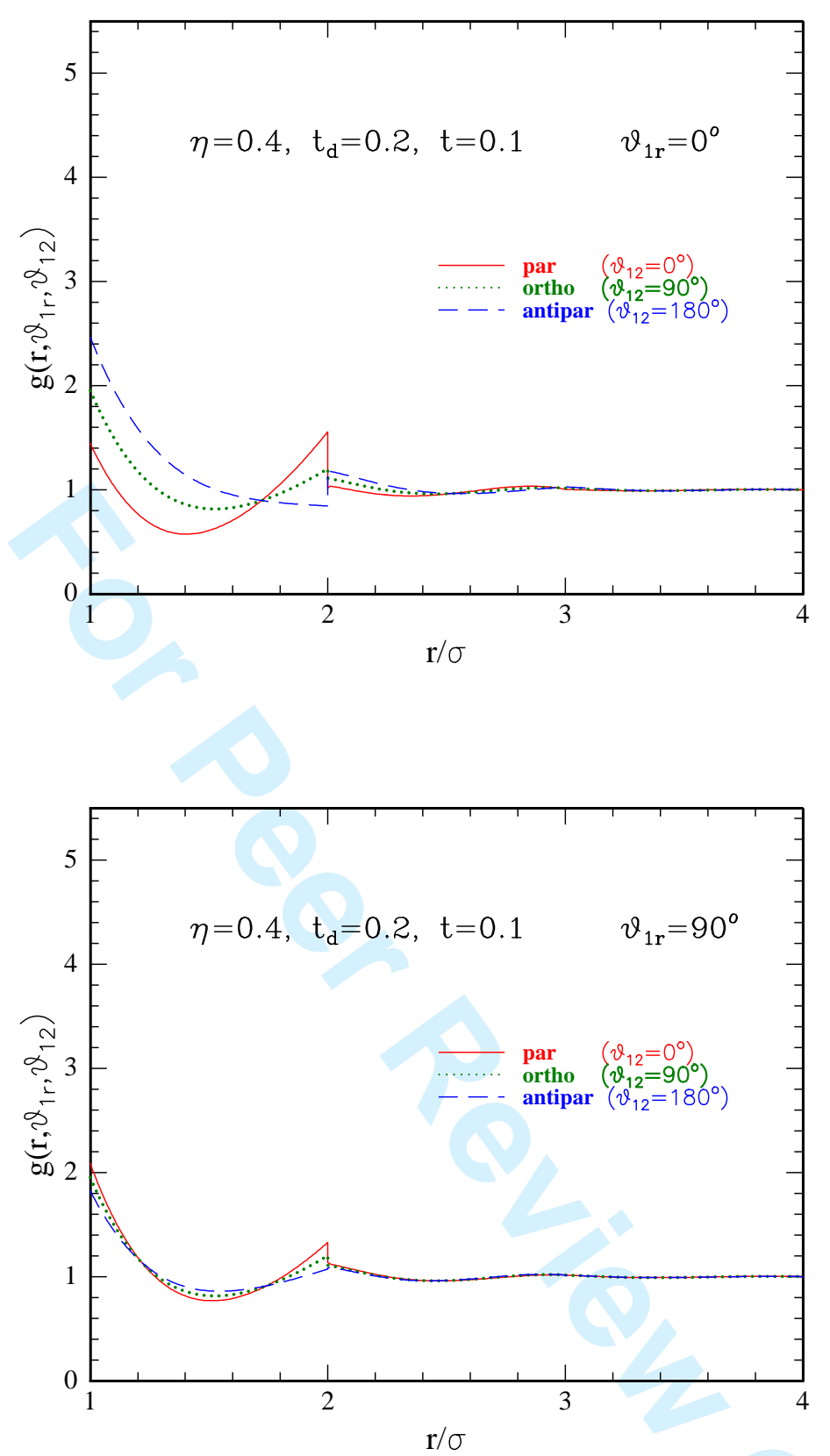

Figure 3. Fluid of hard spheres with dipolar-like patch interaction, plus an isotropic adhesion. Both panels refer to three representative orientations: $\theta_{12}=0^{\circ}, 90^{\circ}$, and $180^{\circ}$ (parallel, orthogonal, and antiparallel), for $\eta=0.4, t_{\mathrm{d}}=0.2$ and $t=0.1$. Upper panel a): regular part of $g_{A}\left(r, \theta_{12}\right) \equiv g\left(r, \theta_{1 r}=0^{\circ}, \theta_{12}\right)$. Lower panel b): regular part of $g_{B}\left(r, \theta_{12}\right) \equiv g\left(r, \theta_{1 r}=90^{\circ}, \theta_{12}\right)$.

With reference to the volume $V_{A B}$, we define the order parameter

$$
\mathcal{P}_{z} \equiv\left\langle\cos \theta_{12}\right\rangle_{V_{A B}}=\frac{1}{V_{A B}} \int_{V_{A B}} d \mathbf{r}_{12}\left\langle\cos \theta_{12} g(1,2)\right\rangle_{\mathbf{u}_{1}, \mathbf{u}_{2}}
$$

where the average, weighted by $g(1,2)$, is performed over all possible positions of the test particle in $V_{A B}$, as well as over all orientations of both molecules 1 and 2 . $\mathcal{P}_{z}$ is proportional to the average z-polarization in the given volume $V_{A B}$ [23].

The usage of this order parameter with $V_{A B}=V_{\text {sphere }}$ is rather common, both for finite $R$ and for $R \rightarrow \infty$. It appears, for instance, in Kirkwood's theory of the 
Figure 4. Three portions of a sphere $S_{R}$ of radius $R$, centred at a generic reference molecule 1 . a) $V_{\text {sphere }}$, coinciding with the whole sphere; b) $V_{\text {forward }}$, conical forward region, defined by a solid angle with $\theta_{A}=0^{\circ}$, $\theta_{B}=60^{\circ}$; c) $V_{\text {lateral }}$, ring-shaped lateral region, with $\theta_{A}=60^{\circ}, \theta_{B}=90^{\circ}$.

dielectric constant [24-26]. On the contrary, its extension with $V_{A B}=V_{\text {forward }}$ or $V_{A B}=V_{\text {lateral }}$ yields two novel indicators with "directional" character $[15,16]$.

The parameter $\left\langle\cos \theta_{12}\right\rangle_{V_{\text {sphere }}}$ with $R \rightarrow \infty$ is also denoted as $\left\langle P_{1}\right\rangle \equiv\left\langle P_{1}\left(\cos \theta_{12}\right)\right\rangle$ and called first-rank orientational order parameter [24], since $P_{1}(x)=x$ is the first-rank Legendre polynomial. In fluids with full orientational disorder one finds $\left\langle P_{1}\right\rangle=0$, whereas $\left\langle P_{1}\right\rangle=1$ occurs only when all dipoles have exactly the same direction.

Since $V_{A B}=\Omega_{A B} R^{3} / 3$ and $\Omega_{A B}=2 \pi\left(\cos \theta_{A}-\cos \theta_{B}\right)$, Eq. (32), coupled with the PY-OL closure, leads to

$$
\left\langle P_{1}\right\rangle_{V_{A B}} \equiv \mathcal{P}_{z}\left(R, \Omega_{A B}\right)=\frac{1}{R^{3}}\left[I_{\Delta}(R)+M_{\Omega_{A B}} I_{D}(R)\right]
$$

where

$$
\begin{gathered}
I_{\Delta}(R)=\int_{\sigma}^{R} d r r^{2} h_{\Delta, \mathrm{reg}}(r)+\Lambda_{\Delta} \\
I_{D}(R)=\int_{\sigma}^{R} d r r^{2} h_{D, \mathrm{reg}}(r)+\Lambda_{D} \\
M_{\Omega_{A B}}=\cos ^{2} \theta_{A}+\cos \theta_{A} \cos \theta_{B}+\cos ^{2} \theta_{B}-1 .
\end{gathered}
$$

Solid angle measures are: $\Omega_{\text {sphere }}=4 \pi$, and $\Omega_{\text {forward }}=\Omega_{\text {lateral }}=\pi$, respectively. While $M_{\text {forward }}=3 / 4$ and $M_{\text {lateral }}=-3 / 4$, one then finds $M_{\text {sphere }}=0$, which implies that $\left\langle P_{1}\right\rangle_{V_{\text {sphere }}}$ simply reduces to $R^{-3} I_{\Delta}(R)$.

For each of the three volumes $V_{\text {forward }}, V_{\text {lateral }}$ and $V_{\text {sphere }}$, Figure 5 shows how $\mathcal{P}_{z}$ varies with increasing the strength $t$ of the isotropic adhesion, at fixed values of $\eta=0.4$ and $t_{\mathrm{d}}=0.2$.

In any case, our three indicators $-\left\langle P_{1}\right\rangle_{V_{\text {forward }}},\left\langle P_{1}\right\rangle_{V_{\text {lateral }}}$ and $\left\langle P_{1}\right\rangle_{V_{\text {sphere }}}$ - all vanish as $R \rightarrow \infty$. This fact means that the orientational order does not concern the whole fluid (as in nematic phases), but is purely local and short-ranged. 

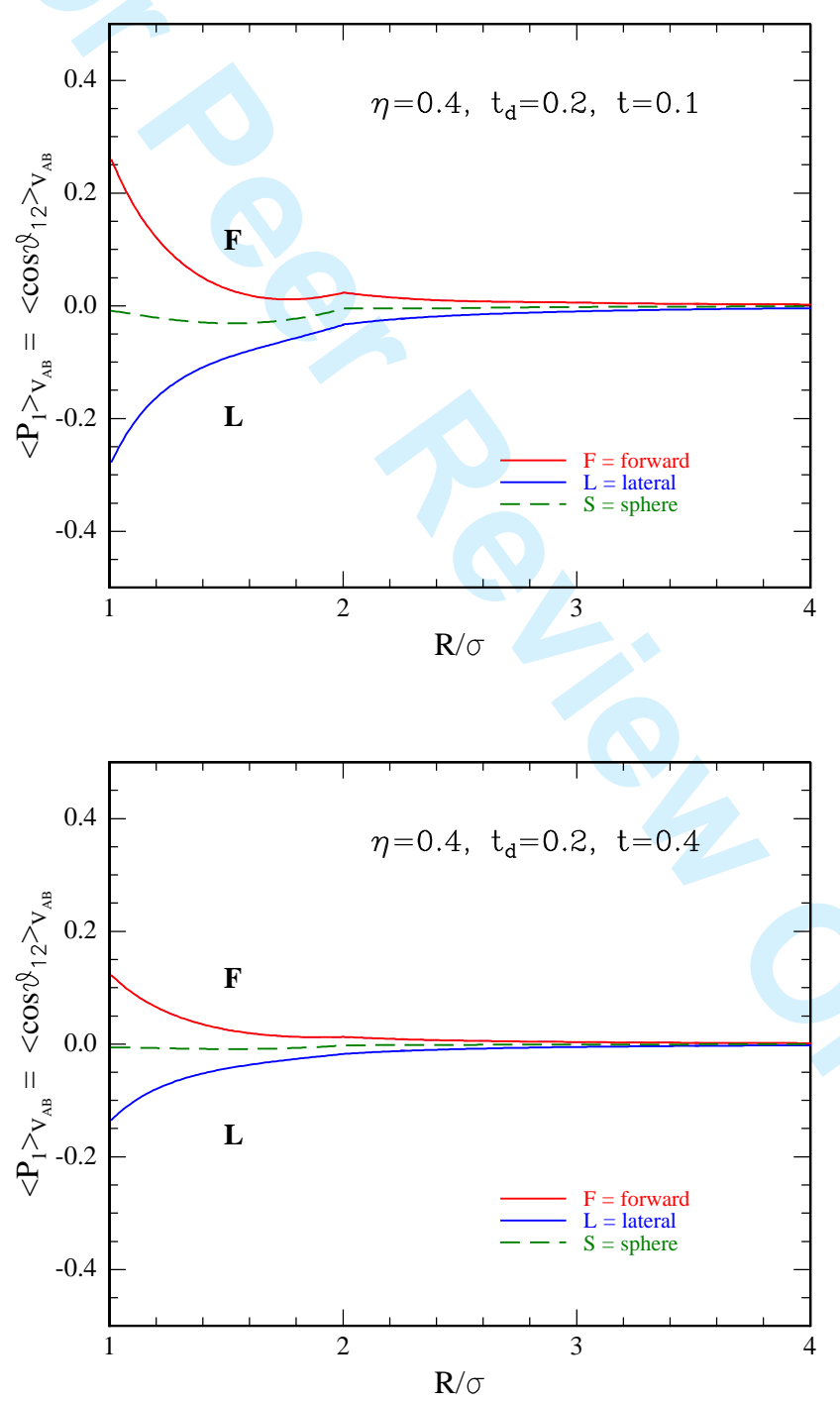

Figure 5. Effect of adding an isotropic adhesion, as shown by the orientational order parameters $\left\langle P_{1}\right\rangle_{V_{A B}}$, related to the average $z$-polarization in the volumes defined by Figure 4 . All panels refer to $\eta=0.4$ and $t_{\mathrm{d}}=0.2$, for the three volumes $V_{\text {forward }}, V_{\text {lateral }}$ and $V_{\text {sphere }}$, respectively. The strength $t$ of the isotropic term increases from the top to the middle, and finally to the bottom panel (plots a), b) and c), respectively). 
Figure 5a) (top panel) refers to the case without isotropic adhesion, $t=0$. Now, $\Lambda_{\Delta}$ vanishes. The large and positive values of $\left\langle P_{1}\right\rangle_{V_{\text {forward }}}$ for $\sigma \leq R \leq 2 \sigma$ indicate the presence of a significant parallel ordering in this space region. Of course, this result stems from an average over both direct contact interactions and indirect ones taking place when $r>\sigma$. The dependence of $\left\langle P_{1}\right\rangle_{V_{\text {forward }}}$ on the radius $R$ of the conical forward region gives some insight into the competition between direct and indirect interactions. In fact, for $R$ close to $\sigma$, the large value of $\left\langle P_{1}\right\rangle_{V_{\text {forward }}} \approx 0.5$ is certainly related to the contact impulsive forces which favour parallel orientations. As $R$ increases, the contribution of indirect interactions favours antiparallel ordering (as shown in Figure 2a), and consequently $\left\langle P_{1}\right\rangle_{V_{\text {forward }}}$ initially decreases. However, when $\sqrt{3} \lesssim R \leq 2 \sigma$ parallel orientations become again more probable, so that $\left\langle P_{1}\right\rangle_{V_{\text {forward }}}$ starts to increase. The behaviour for $R>2 \sigma$ can be explained in a similar way.

Figure 5a) also shows that antiparallel ordering prevails in $V_{\text {lateral }}$ with $\sigma \leq$ $R \leq 2 \sigma$, as indicated by the large and negative values of $\left\langle P_{1}\right\rangle_{V_{\text {lateral }}}$. On the other hand, $\left\langle P_{1}\right\rangle_{V_{\text {sphere }}}$ exhibits rather small values, leading to conclude that no significant orientational order exists in $V_{\text {sphere }}$. In other words, the presence of particles with different orientations near the polar and the equatorial regions of molecule 1 is completely hidden by the average involved in $\left\langle P_{1}\right\rangle_{V_{\text {sphere }}}$, due to the symmetry of the spherical volume. On the contrary, the introduction of anisotropic indicators, such as $V_{\text {forward }}$ and $V_{\text {lateral }}$, based upon asymmetric volumes, allows to get a more detailed picture of the microstructure.

Figures 5b) and 5c) (middle and bottom panels, respectively) illustrate the effect of adding an isotropic sticky attraction to HS particles with dipolar-like patch. We have plotted the results only for two cases, i.e. $t=0.1$ and $t=0.2$. In the former case, $t=0.5 t_{\mathrm{d}}$, an apparent reduction of the orientational order is found in both the forward and lateral volumes. Such an orientational randomization is even more enhanced in the latter case, $t=2 t_{\mathrm{d}}$, where all $\left\langle P_{1}\right\rangle_{V_{A B}}$ are nearly vanishing.

These results are fully reasonable. As already observed, an isotropic surface attraction allows two molecules to adhere, whatever their orientations may be. This behaviour strongly contrasts with the tendency to stick only when specific portions of the molecular surfaces are facing each other.

\section{Concluding remarks}

DHSs, which represent the simplest model for fluids with dipolar interactions, have been widely investigated in the literature by means of both integral equations and computer simulations [23, 27-30]. Such studies show that, at low temperatures, hard spheres with long-ranged purely dipolar interactions exhibit: at low densities, a strong tendency to form chain-like structures, with head-to-tail alignment; at high densities, a transition to a ferroelectric fluid state. Furthermore, by switching on an additional isotropic attraction gradually (i.e. a Lennard-Jones term as in the Stockmayer fluid [23], or a Yukawa tail [30]), there is a changeover from dipole chaining to condensation. In other words, increasing the isotropic attraction disfavours structures where particles have 1 or 2 bonded neighbours (pairs or linear chains), but favours clustering with particles having 3 or more neighbours (branched chains or more compact globules, which act as condensation seeds) [30, 31].

Our work has shown that the DP and DP + models strongly differ from the DHS one, since they consider only surface interactions. In fact, the dipolar-like patch is obtained by truncating the long-ranged DHS potential exactly at contact. As a consequence, our models are strictly short-ranged (more precisely, the DP 
interaction has null width at $r=\sigma$, being modeled by $\delta(r-\sigma)$ terms). Thus the comparison between DP and DHS models has been interesting, since it has somehow shown the effect of varying the range of the dipolar interaction drastically.

The local orientational order of the DP fluid - with its alternance of parallel and antiparallel preferred orientations - seems to be the most important finding, which strongly distinguishes our models from the DHS one. On the other hand, the effect of adding an isotropic adhesion is essentially similar to the long-ranged case: just as for the Stockmayer model, the isotropic attraction disrupts the chain ordering, in favour of the formation of more spherical condensation aggregates.

Our calculations have been performed only for a rather small set of parameter values. In fact, the main limitation of the present study consists in the orientational linearization of the PY-OL closure. This approximation allows to obtain analytic solutions for both the DP and DP+ models, but restricts the possible values of the interaction strengths $t_{\mathrm{d}}$ and $t$. Consequently, the PY-OL solutions can hardly be applied to high coupling regimes, where the most important phenomena should occur. Nevertheless, our first qualitative results seem to confirm the importance of the interaction range in determining the kind of microstructure, and suggest the opportunity of investigating similar models with more realistic potentials.

To conclude this paper, we report a last useful information. At the end of the present work, it has been published an interesting paper on a realistic system which has strong similarities with our model and thus provides it with more physical plausibility. It deals with a fluid of charged colloidal spheres, whose surface is decorated with a thin and nonuniform layer of adsorbed polyelectrolytes [32]. On varying the ionic strength of the electrolyte solution, the screening length $\kappa_{\mathrm{D}}^{-1}$ can become short compared to the size of the colloidal particles. In this case, a "patch attraction" between like-charged complexes is observed, due to the asymmetric surface distribution of the mobile charges, which can be represented by defining dipole moments of the complexes.

Surprisingly enough, those authors found that "the eventual aggregation of the two complexes occurs with the antiparallel orientation of the dipoles" and remark that "this antiparallel orientation of the dipole doublet signals the absence of a longrange dipole-dipole attractive contribution to the mean-force interparticle potential [32]." All this qualitatively agrees with our work.

\section{Acknowledgements}

The support of the PRIN-COFIN under Grant No. 2007B58EAB is gratefully aknowledged.

\section{References}

[1]F. Sciortino, P. Tartaglia, and E. Zaccarelli, J. Phys. Chem. B 109, 21942 (2005).

[2]E. Bianchi, J. Largo, P. Tartaglia, E. Zaccarelli, and F. Sciortino, Phys. Rev. Lett. 97, 168301 (2006).

[3] A. Lomakin, N. Asherie, and G. B. Benedek, Proc. Natl. Acad. Sci. USA 96, 9645 (1999).

[4]F. W. Starr, and J. F. Douglas, J. Chem. Phys. 119, 1777 (2003).

[5]Z. Zhang, and S. C. Glotzer, Nano Lett. 4, 1407 (2004).

[6]S. C. Glotzer, Science 306, 419 (2004).

[7]F. Sciortino, E. Bianchi, J. F. Douglas, and P. Tartaglia, J. Chem. Phys. 126, 194903 (2007).

[8] G. Jackson, W. G. Chapman, and K. E. Gubbins, Mol. Phys. 65, 1 (1988).

[9] E. Mileva, and G. T. Evans, J. Chem. Phys. 113, 3766 (2000).

[10]N. Kern, and D. Frenkel, J. Chem. Phys. 118, 9882 (2003).

[11]R. Fantoni, D. Gazzillo, A. Giacometti, M.A. Miller and G. Pastore, J. Chem. Phys. 127, 234507 (2007). 


\section{Page 17 of 17

[12] A. Giacometti, F. Lado, J. Largo, G. Pastore, and F. Sciortino, J. Chem. Phys. 131, 174114 (2009).

[13] A. Giacometti, F. Lado, J. Largo, G. Pastore, and F. Sciortino, J. Chem. Phys. 132, 174110 (2010).

[14]D. Gazzillo, R. Fantoni, and A. Giacometti, Phys. Rev. E 78, 021201 (2008).

15]D. Gazzillo, R. Fantoni, and A. Giacometti, Phys. Rev. E 80, 061207 (2009).

[16]D. Gazzillo, J. Chem. Phys. 133, 034511 (2010).

[17] J. P. Hansen, and I. R. McDonald, Theory of Simple Liquids, 3rd Ed. (Academic Press, Amsterdam, 2006).

[18]M. S. Wertheim, J. Chem. Phys. 55, 4291 (1971).

[19]R. J. Baxter, J. Chem. Phys. 49, 2770 (1968).

[20] R. J. Baxter, in Physical Chemistry, an Advanced Treatise, Vol. 8A, ed. by D. Henderson (Academic, New York, 1971) Chap. 4.

[21] G. A. Chapela, and E. Martina, Mol. Phys. 55, 97 (1985).

[22]C. G. Joslin, and C. G. Gray, Mol. Phys. 57, 1197 (1986).

[23]S. Klapp, and F. Forstmann, J. Chem. Phys. 106, 9742 (2004).

[24] C. G. Gray, and K. E. Gubbins, Theory of Molecular Fluids, Vol. I (Clarendon Press, Oxford, 1984).

[25] V. I. Kalikmanov, Statistical Physics of Fluids (Springer, Berlin, 2001).

[26] M. Valiskó, and D. Boda, J. Phys. Chem. B 109, 6355 (2005).

[27]D. Levesque, and J. J. Weis, Phys. Rev. E 49, 5131 (1994).

[28] G. Ganzenmüller and P.J. Camp, J. Chem. Phys. 126, 191104 (2007).

[29] N. G. Almarza, E. Lomba, C. Martin, and A. Gallardo, J. Chem. Phys. 129, 234504 (2008).

[30] G. Ganzenmüller, G. N. Patey, and P. J. Camp, Mol. Phys. 107, 403 (2009).

[31]T. Tlusty and S.A. Safran, Science 290, 1328 (2000).

[32]D. Truzzolillo, F. Bordi, F. Sciortino, and S. Sennato, J. Chem. Phys. 133, 024901 (2010). 